\title{
Revisión sistemática de bibliografía sobre traducción audiovisual y estudios LGBTQ+ (2000-2020): dimensiones epistemológica y metodológica
}

\section{A systematic literature review on audiovisual translation studies and LGBTQ+ studies + (2000-2020): epistemological and methodological dimensions}

\author{
IVÁN VILLANUEVA JORDÁN \\ ivan.villanueva@upc.pe \\ Universidad Peruana de Ciencias Aplicadas \\ Frederic Chaume VARELA \\ chaume@uji.es \\ Universidad Jaume I
}

Fecha de recepción: 08/03/2021

Fecha de aceptación: 15/07/2021

Resumen: El artículo presenta y discute los resultados de una revisión sistemática de bibliografía sobre estudios de traducción audiovisual y estudios LGBTQ+. Según el modelo de metasíntesis, se han reunido 28 estudios empíricos publicados entre 2000 y 2020. Los ejes del análisis de dichas fuentes han sido epistemológicos y metodológicos. Los resultados de la sistematización permiten conocer los supuestos de base en torno a la visión del mundo (pospositivista, constructivista o transformadora) del que parten las investigaciones. Asimismo, revelan oportunidades de mejora en el diseño metodológico considerando que los estudios de caso son una estrategia recurrente en las investigaciones. El propósito de esta metasíntesis es proponer un diagnóstico sobre la intersección entre dos miradas teóricas (TAV y LGBTQ+), que constituyen un campo de investigación en crecimiento constante.

Palabras clave: Traducción audiovisual, LGBTQ+, Estudios de género, Metasíntesis, Estado de la cuestión.

Abstract: This article presents and discusses the results of a systematic literature review on audiovisual translation studies and LGBTQ+ studies. Using metasynthesis as a qualitative research method, 28 empirical studies published between 2000 and 2020 have been collected. The analysis of these sources has focused on epistemological and methodological aspects. The results of the systematization reveal the underlying assumptions about 
the researchers' worldview (post-positivist, constructivist or transformative). The results also show opportunities for improving the methodological design, considering that case studies are a common method in these sources. The purpose of this meta synthesis is to propose a diagnosis of the intersection between two theoretical perspectives (AVT and LGBTQ+), which today are a field of research in constant growth.

Keywords: Audiovisual translation, LGBTQ+, Gender studies, Metasynthesis, Literature review.

\section{INTRODUCCIÓN}

La metodología de la investigación integra los supuestos, postulados, lineamientos y técnicas de producción de datos elegidos por el investigador - la investigadora para que otros analicen su proceso investigativo, lo critiquen, repliquen o adapten a un nuevo contexto u otro objeto de estudio (Schensul, 2008, p. 516). En el campo de la traductología, según Rojo (2013), la metodología y los diseños metodológicos han cambiado de acuerdo con los paradigmas imperantes que sucedieron tras la configuración disciplinar de la traducción como campo de estudio hacia la mitad del siglo XX. La consolidación de la disciplina, así como de otras vertientes que han despegado a partir de modelos iniciales exigen una mirada diagnóstica constante para reconocer las «fortalezas y debilidades» del trabajo investigativo (Rojo, 2018). Ello se debe a que la metodología de la investigación no es un área estática o fácil de compartimentalizar, ya sea mediante criterios cronológicos o según el tipo de datos de la investigación. Se trata, más bien, de modelos dinámicos que van reorganizándose de acuerdo con los modelos teóricos sobre la traducción que subyacen a toda investigación (Chesterman \& Williams, 2002, p. 48) o a la dinámica entre el régimen de verdad y el método.

Con respecto a la investigación en traducción audiovisual (TAV), cuya consolidación como campo de estudio resulta innegable desde el inicio del siglo XXI (Chaume, 2018), los Estudios Descriptivos de Traducción (EDT) han provisto un camino metodológico que ha sido recorrido en contextos nacionales diversos (Karamitroglou, 2000; Pedersen, 2011), aplicados a distintos géneros televisuales o fílmicos (Martí Ferriol, 2010; Martínez Sierra, 2008) o a categorías de análisis específicas, por ejemplo, los referentes culturales (Ranzato, 2016), con la finalidad de abstraer normas de traducción en relación con un corpus ad hoc en estudios de caso, aunque también mediante el diseño de corpus explotables mediante distintas categorías de análisis (Freddi, 2012; Valentini, 2013). Chaume (2018) identifica, en el desarrollo de los EDT en la TAV, el surgimiento de enfoques sociológicos; sin embargo, también llama la atención sobre el 
impacto de la tecnología en campos temáticos y diseños metodológicos novedosos, por ejemplo, en relación con los estudios cognitivos.

El presente estudio tiene como finalidad explorar las características metodológicas de investigaciones sobre TAV y estudios LGBTQ+ publicadas entre los años 2000 y 2020 , y que constituyen un corpus de 28 publicaciones entre capítulos de libros y artículos de revistas especializadas. En relación con el balance elaborado por Chaume (2018), la presente sistematización de la literatura científica pretende ser una mirada más cercana sobre investigaciones que podrían ubicarse en lo que el autor denomina "case studies with a cultural approach» (p. 55). Centrar la visión en estos estudios permite explorar los supuestos subyacentes entre metodología y epistemología de dichos estudios, así como la manera en que se comprenden los casos de estudio, el valor interpretativo de los datos y la capacidad de transferencia o generalización teórica. Los resultados de esta exploración metateórica podrán ser de utilidad en el diseño metodológico de próximas investigaciones sobre TAV, pero también en estudios relacionados con la diversidad sexual y de género. En una reciente reseña de obras sobre estudios LGBTQ+ en la traductología, Attig (2020) ha identificado exclusiones recurrentes en la literatura académica contemporánea que derivan de caer en el binarismo hétero/homo. Como se explicará en las siguientes secciones, dicho tipo de sesgos no obedecen solo a características del objeto de estudio, sino a los supuestos fundamentales sobre la realidad, la traducción o la sexualidad.

En la siguiente sección se explica brevemente el modelo de análisis sistemático de la literatura denominado «metasíntesis»; asimismo, se exponen las características del diseño de esta revisión de la literatura en particular. Más adelante se presentan los resultados de la metasíntesis en torno a ejes temáticos como la visión del mundo, el enfoque metodológico, el tipo de datos y el diseño de los estudios de caso. Sin dudar de las implicaciones e innovaciones en el conocimiento de la TAV y la representación de las identidades sexuales y de género de los estudios analizados, el propósito diagnóstico de una revisión sistemática de la literatura es valorar lo avanzado y reconocer las oportunidades de mejora. $Y$ ese es el propósito general del presente artículo.

\section{LA METASÍNTESIS COMO REVISIÓN SISTEMÁTICA DE LA BIBLIOGRAFÍA}

Una metasíntesis permite relacionar los resultados o características particulares de un estudio con aquellos de otro estudio. Establecer estos vínculos entre investigaciones requiere un trabajo analítico y, por lo general, crítico por parte del investigador, que debe relacionarse con la información propuesta en dichas investigaciones y aceptarlas, valorarlas o rechazarlas 
como conocimiento. Los vínculos entre relaciones se han denominado, en inglés, translations, como explican a continuación Onwuegbuzie y Frels (2016):

A meta-synthesis involves translating the findings of one study into another study. These translations lie in a translation continuum, with one end of the continuum representing studies that can be translated using metaphors, concepts, and themes that are common to both -or what can be referred to as reciprocal translations. At the other end of the translation continuum are studies that contain findings that are in opposition to each other, or that represent contradictions or paradoxes-or what can be referred to as refutational translations (cf. Noblit \& Hare, 1988). In between these two poles are studies overlap without being substitutional -or what can be referred to as overlapping translations (p. 24).

La metasíntesis como revisión sistemática de la literatura sobre un tema o campo específico requiere un proceso reflexivo y documentado que permita dar cuenta de las distintas fases del análisis, desde las activididades de búsqueda de información, pasando por los criterios de selección y exclusión de las fuentes, hasta las categorías de síntesis de los datos. Onwuegbuzie y Frels (2016) proponen un modelo trifásico que se subdivide en siete pasos, como se aprecia en la figura 1. A continuación, se expondrá el diseño de la metasíntesis objeto del artículo según las fases propuestas por los autores. 


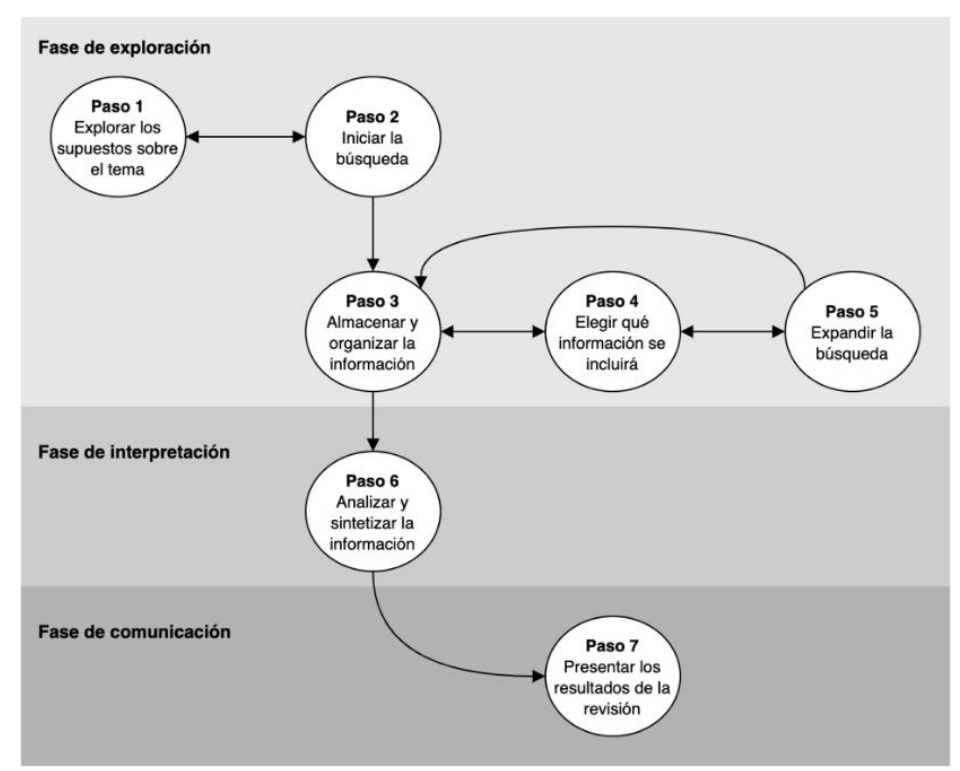

Figura 1. Pasos de la revisión integral de la literatura Fuente: adaptado de Onwuegbuzie \& Frels (2016)

\subsection{Fase de exploración}

El diseño de esta metasíntesis se ha trazado considerando la manera en que los estudios de género han calado progresivamente en las investigaciones sobre traducción audiovisual, lo que constituye actualmente una mirada investigativa consolidada (de Marco, 2012). A esta perspectiva de género se suman los estudios gais y la contribución desde la teoría queer en la problematización de las representaciones de las identidades de género y sexuales disidentes (Martínez Pleguezuelos, 2018). A partir de estos supuestos, se estableció una fuente de objetivos procedimentales y analíticos que guiaron la metasíntesis.

Una vez planteados los objetivos de la revisión de literatura, la recolección de fuentes se basó en distintas estrategias de búsqueda en bases de datos académicas, que se señalan en la siguiente figura. 
Fase de exploración

Paso 2. Iniciar la búsqueda

Estrategias de búsqueda

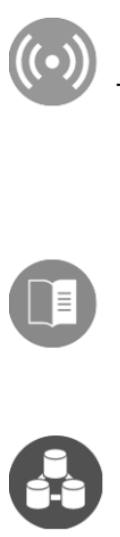

Bola de nieve

Se buscaron artículos que citaban textos

fundacionales en el estudio de las

identidades gais en la traductología

161 fuentes (universo textual)

Bibliografías

especializadas

Bases de datos

Google Scholar, Scopus, Web of Science,

BITRA, Taylor \& Francis; John Benjamins y

Wiley Online Library

Figura 2. Estrategias de búsqueda

Fuente: elaboración propia

Sobre la estrategia de bola de nieve, los estudios que sirvieron para identificar otras fuentes relevantes fueron los trabajos de Harvey (1998, 2000a). En cuanto a las bibliografías especializadas se revisaron las fuentes reunidas por Baer y Kaindl (2015) para el congreso Queering Translation, Translating the Queer. En cuanto a las bases de datos, se realizaron búsquedas con las palabras «translation», «audiovisual translation», «queer», «gay» en las bases de datos Google Scholar, Scopus, Web of Science, BITRA (Franco Aixelá, 2020) y las de las revistas especializadas de Taylor \& Francis; John Benjamins y Wiley Online Library. El resultado de esta búsqueda fueron 161 fuentes entre artículos, capítulos de libros, actas de congresos y trabajos de fin de máster y tesis.

A este conjunto general de fuentes se aplicaron los siguientes criterios de inclusión. 


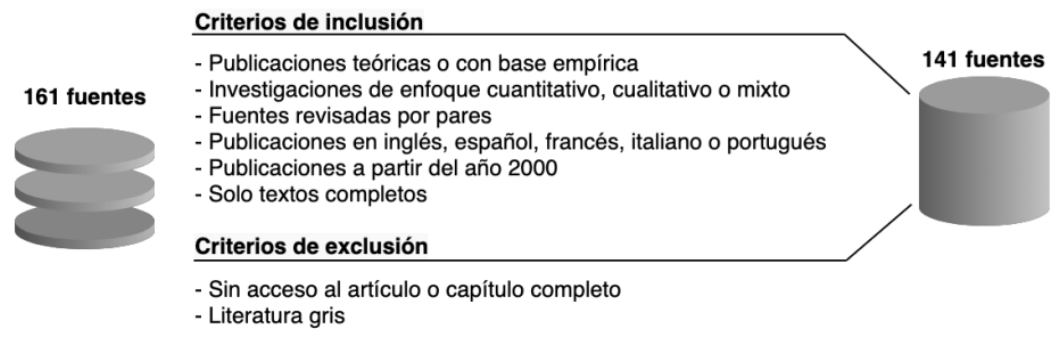

Figura 3. Criterios de inclusión y exclusión Fuente: elaboración propia

De esta manera quedaron un total de 141 fuentes: 76 artículos de revistas, 63 capítulos de libros monográficos (que incluyen las introducciones) y dos (2) libros. Las fuentes se categorizaron adicionalmente considerando si se basaban en investigaciones empíricas o si eran artículos o capítulos teóricos (por ejemplo, estados de la cuestión, ensayos académicos, textos conceptuales, experiencias docentes, entre otros) que luego se excluyeron.

Los estudios empíricos a su vez se clasificaron de acuerdo con el tipo de modalidad de traducción (escrita o audiovisual) y al medio (cine, televisión, internet). Después de esta organización, se identificaron 28 investigaciones empíricas sobre TAV y estudios LGBTQ+ que componen el corpus específico de la metasíntesis.

\subsection{Fase de interpretación}

La fase de interpretación incluye trabajo analítico y tareas de clasificación y categorización de las fuentes. Cada fuente se leyó al menos dos veces. Los documentos se gestionaron mediante el programa Nvivo, que a su vez facilitó el marcado, codificación y recuperación de pasajes relevantes relacionados con las categorías que fueron emergiendo durante la lectura. De esta manera, se establecieron relaciones (translations, como se explicó antes) de similitud, oposición o complementariedad entre los estudios, y se produjo la síntesis de ideas en torno a las categorías señaladas en la siguiente tabla:

\begin{tabular}{|lll|}
\hline & \multicolumn{1}{|c|}{ Categoría } & \multicolumn{1}{c|}{ Detalles } \\
\hline 1 & Visión del mundo & $\begin{array}{l}\text { constructivista, pospositivista, transformadora, } \\
\text { crítica }\end{array}$ \\
2 & Enfoque & $\begin{array}{l}\text { cualitativo, cuantitativo, mixto } \\
\text { estudio de caso, estudio de corpus, análisis de } \\
\text { contenidos, entre otros }\end{array}$ \\
\hline
\end{tabular}

Hikma 20 (2) (2021), 95 - 126 


\begin{tabular}{|c|c|c|}
\hline 4 & $\begin{array}{l}\text { Estudio de caso como } \\
\text { estrategia metodológica }\end{array}$ & $\begin{array}{l}\text { Se enuncia que es un estudio de caso de manera } \\
\text { explícita o se debe inferir. }\end{array}$ \\
\hline 5 & Calidad del caso elegido & crítico, atípico, típico \\
\hline 6 & Lenguas & lenguas de los textos analizados \\
\hline 7 & Tipo de datos & $\begin{array}{l}\text { cualitativos (solo lingüísticos, multimodales), } \\
\text { cuantitativos }\end{array}$ \\
\hline 8 & Modalidad de TAV & doblaje, subtitulación, entre otras \\
\hline 9 & Medio & televisión, cine, internet \\
\hline 10 & $\begin{array}{l}\text { Conceptualización de } \\
\text { TAV }\end{array}$ & $\begin{array}{l}\text { traducción subordinada, transcreación, } \\
\text { localización, entre otras }\end{array}$ \\
\hline 11 & Tipo de análisis & $\begin{array}{l}\text { análisis textual-contrastivo, multimodal, } \\
\text { narratológico, entre otros }\end{array}$ \\
\hline 12 & $\begin{array}{l}\text { Relación } \\
\text { instrumento/restricciones }\end{array}$ & $\begin{array}{l}\text { los instrumentos incluyen datos sobre restricciones } \\
\text { semióticas o formales }\end{array}$ \\
\hline 13 & Caso & $\begin{array}{l}\text { título del producto o productos audiovisuales } \\
\text { analizados }\end{array}$ \\
\hline 14 & $\begin{array}{l}\text { Diseño según número } \\
\text { de casos }\end{array}$ & caso único, casos múltiples \\
\hline 15 & $\begin{array}{l}\text { Conceptualización } \\
\text { sexualidad/género }\end{array}$ & $\begin{array}{l}\text { Se omiten o se exponen supuestos teóricos sobre } \\
\text { las identidades de género o sexuales o la noción } \\
\text { de sexo género. }\end{array}$ \\
\hline
\end{tabular}

Tabla 1 : Categorías analíticas de la metasíntesis TAV-LGBTQ+ Fuente: elaboración propia

Como se muestra en las imágenes 4 y 5 , las fuentes sobre TAV tienen un par de características de que se deben señalar de partida. En primer lugar, 8 fuentes (29\% de los estudios) se publicaron entre los $2000 \mathrm{y}$ 2010, mientras que $20(71 \%)$ se publicaron durante la década siguiente. Por otro lado, más de la mitad de las fuentes (15 estudios, $54 \%$ ) son sobre productos televisuales, mientras que un $32 \%$ (9 estudios) sobre películas para cine y solo $14 \%$ (4 estudios) abordan casos de internet. Ambos hechos sobresalientes, el periodo de producción y la producción de series de televisión, resultan comprensibles si se considera el proceso de hipervisibilidad de la diversidad sexual y de género en los medios de comunicación y en particular en la televisión desde inicios del siglo XXI. Al respecto, Pidduck (2011) ha señalado que lo híper- de esta forma de visibilidad revela un exceso en la representación de identidades que antes eran innombrables. Este exceso de representaciones (cantidad), pero también la desmesura de lo representado (calidad) se basa en una intención constante de domesticar la diferencia. 


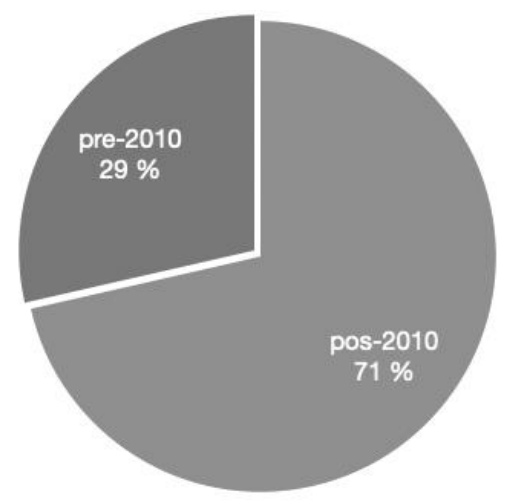

Figura 4. Periodo de publicación Fuente: elaboración propia

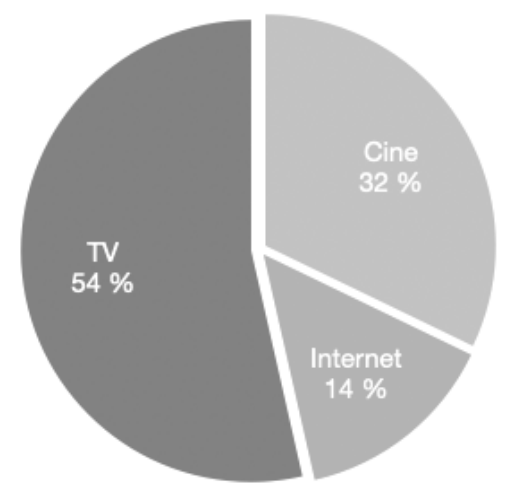

Figura 5. Medio

Fuente: elaboración propia

Otro factor importante de los estudios recogidos para esta metasíntesis es la distribución según pares de lenguas (figura 6). Los estudios más numerosos son los que trabajan con la combinación italianoinglés ( $36 \%, 10$ estudios) y estos abordan casi exclusivamente el doblaje; mientras que los estudios sobre el español y el inglés alcanzan un $29 \%$ (8 estudios: 5 sobre doblaje y 3 sobre subtítulos). En el caso de la combinación francés-inglés, son solo 3 estudios y 2 de estos son casos de adaptaciones de películas francesas mediante producciones norteamericanas (este tema se abordará con detalle en la sección 2.6). Por otro lado, son 3 estudios los 
que trabajan con la combinación chino-inglés (Bowman, 2010; Jiang, 2020; Guo \& Evans, 2020), 1 estudio sobre el par indonesio-inglés (Boellstorff, 2003) y 1 estudio sobre el par griego-inglés (Asimakoulas, 2012). Un estudio incluye análisis contrastivos entre varias lenguas con el inglés (Lewis, 2010), mientras que otro aborda procesos de traducción mediante una lengua franca (Leung, 2016).

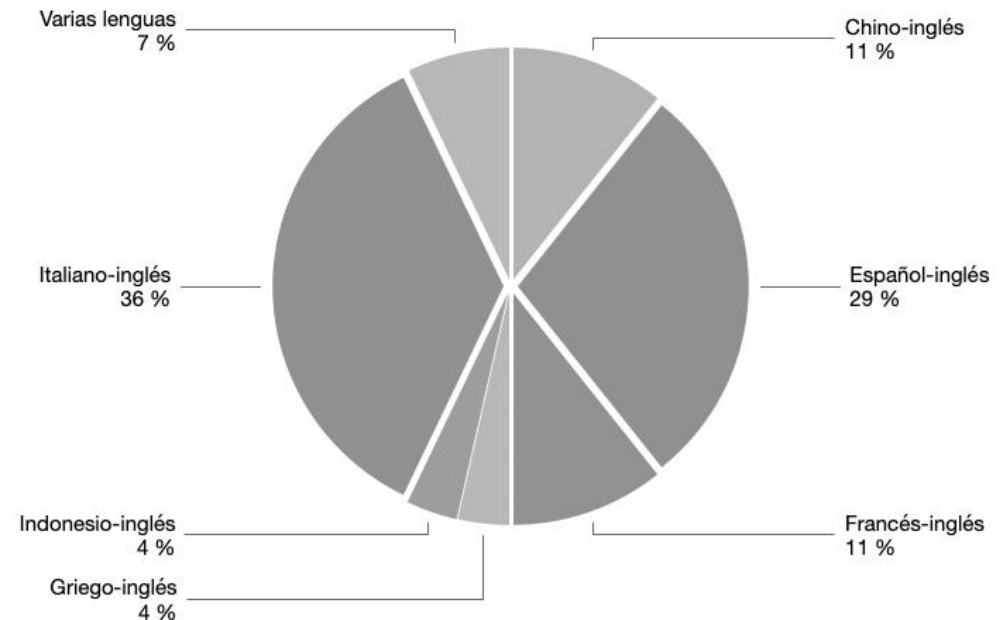

Figura 6. Lenguas de traducción de los estudios de caso Fuente: elaboración propia

A partir de las categorías de análisis (tabla 1), se organizó la fase de comunicación mediante géneros académicos, en particular, artículos de investigación. El presente artículo recoge así las categorías analíticas vinculadas con aspectos epistemológicos, del enfoque y diseño metodológico, y los tipos de datos usados en las investigaciones publicadas sistematizadas.

\section{VISIÓN DEL MUNDO Y ENFOQUE METODOLÓGICO}

El concepto de visión del mundo (worldview) proviene de la propuesta metodológica de Creswell y Creswell $(2018)^{1}$, quienes establecen cuatro

\footnotetext{
${ }^{1}$ We have chosen to use the term worldview as meaning "a basic set of beliefs that guide action" (Guba, 1990, p. 17). Others have called them paradigms (Lincoln, Lynham, \& Guba, 2011; Mertens, 2010); epistemologies and ontologies (Crotty, 1998), or broadly conceived research methodologies (Neuman, 2009). We see worldviews as a general philosophical orientation about the world and the nature of research that a researcher brings to a study. Individuals develop worldviews based on their discipline orientations and research communities,
} 
paradigmas: el pospositivista, el constructivista, el transformador y el pragmático. En un registro epistemológico, el pospositivismo y el constructivismo tienen menos puntos de encuentro, mientras que la visión del mundo transformadora es mucho más próxima al constructivismo. El paradigma pospositivista halla su fundamento en relaciones de determinación entre una causa y su consecuencia. Por ello, los diseños de investigación de este tipo recurren a planteamientos iniciales o hipótesis que establecen una serie de variables como determinantes de otros fenómenos. Como reconocen Angelelli y Baer, esta manera de concebir el mundo "the positivist illusion of capturing the one true meaning of an utterance» (Angelelli \& Baer, 2016, p. 7)- se encuentra vigente sobre todo en el ámbito profesional de la traducción y la interpretación. Por su parte, el pragmatismo establece que la verdad se alcanza mediante la práctica y el método, con lo que se aleja de las bases epistemológicas del positivismo y el constructivismo que privilegian la reflexión y la práctica razonada antes que la acción (MsCaslin, 2008).

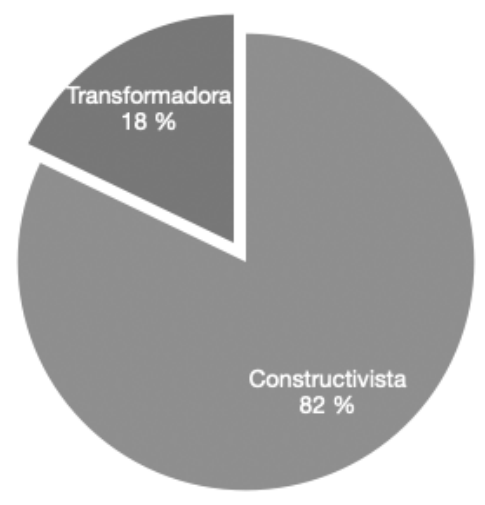

Figura 7. Visión del mundo Fuente: elaboración propia

Como se aprecia en la figura 7, en los estudios analizados se pueden identificar dos visiones del mundo: la constructivista en $82 \%$ (23) de estudios y la transformadora en $18 \%$ (5). El paradigma constructivista se hace patente cuando la TAV se comprende como un medio de creación y circulación de representaciones. El trabajo de representación, definido con muchísima claridad por Hall (1997), implica comprender que las personas

advisors and mentors, and past research experiences. The types of beliefs held by individual researchers based on these factors will often lead to embracing a strong qualitative, quantitative, or mixed methods approach in their research. (Creswell \& Creswell, 2018)

Hikma 20 (2) (2021), 95 - 126 
crean significados mediante el lenguaje, pero también mediante otras prácticas y procesos simbólicos (p. 11). Así entendida, la función del lenguaje, de las lenguas y de la traducción es representacional, en la que se crean y recrean significados relacionados con distintos ámbitos de la experiencia humana, la sexualidad y el género. La TAV se comprende, en relación con las identidades sexuales disidentes, como un práctica que puede perpetuar estereotipos de género (Díaz Pérez, 2018; Ranzato, 2012; Turek, 2012), revelar las manipulaciones ideológicas a las que distintos sistemas culturales son más propensos (Ranzato, 2015), pero también como un medio para el cambio social con el que se puede lograr la integración de grupos minoritarios (Valdeón, 2010). En otras palabras, pero sin dejar la mirada socioconstructivista, Martínez Pleguezuelos (2017) propone lo siguiente:

\begin{abstract}
[C]ualquier texto traducido que tenga una temática LGTB puede llegar a convertirse en un barómetro mediante el cual analizar qué posición ocupan los factores sociales y culturales en la afirmación y recepción de todas aquellas sexualidades disidentes en una sociedad dada (p. 37).
\end{abstract}

Una visión del mundo transformadora conlleva investigaciones con fines de emancipación de prácticas y conocimiento vinculado con posiciones de poder ilegítimas. En estos casos, los investigadores revelan su posición crítica frente al conocimiento o ponen de relieve la posición de los sujetos participantes de un fenómeno estudiado. La contribución de la investigación resulta de las formas alternativas de comprender la realidad o de actuar en esta, ya sea mediante nuevas categorías teóricas críticas o mediante prácticas 0 hechos transgresores existentes. La investigación de Jiang (2020), sobre el drama para televisión The Story of Yanxi Palace (Hui Kaidong y Wen Deguang, 2018), es un claro ejemplo sobre cómo las acciones de los sujetos son fundamentales para la construcción de conocimiento: "Empowered by the anti-official carnival notion, gay fans' retranslations challenge the status of dominant regimes of knowledge/power and can be a resistance to an ideological hierarchy which places normative interest and attention over» (Jiang, 2020, p. 14). Asimismo, Bassi (2017, 2018) también focaliza a los sujetos mientras se señala la importancia política de sus acciones:

Through this translation work carried out by «invisible» volunteer translators, dominant discourses on sexuality and subjectivity in late capitalism are disassembled and reconstituted in surprising ways. Thus, the stakes in rendering this kind of translation work visible lie in the political possibilities that it opens up for research on both volunteer translation and in queer studies. In fact, it is the affective and cognitive labor of the international users of this online

Hikma 20 (2) (2021), 95 - 126 
platform that permitted the internationalization of this anti-bullying online campaign (Bassi, 2017, p. 67; el subrayado es propio).

Como se puede ver, el posicionamiento de la autora apunta a un cambio en las dinámicas sociales y a la manera en que los sujetos pueden alcanzar su emancipación mediante la crítica de las estructuras sociales y de poder.

En otro caso de visión transformadora, Lewis (2010) parte de los argumentos emancipatorios de la traducción poscolonial y la traducción feminista, en relación con la hegemonía colonizadora y patriarcal, respectivamente, para proponer que la traducción queer sea un medio de resistencia contra la heteronormatividad. La autora propone una revisión del reconocido mapa de la traductología (Translation Studies) delineado inicialmente por James S. Holmes para incluir una mirada queer en cada una de sus ramas. Por ejemplo, en el caso de los estudios aplicados, según Lewis (2010), su propósito sería el de «train translators to recognise queer elements in texts and to use creative strategies to preserve those elements in translation, include translator criticism, and create resources to aid translators in translating queer texts» (p. 8).

Las visiones del mundo constructivistas y transformadoras son recurrentes en los estudios que integran categorías teóricas como el género, las identidades sexuales o lo queer. Estas visiones del mundo van de la mano de teorías de la traducción posestructuralistas que dejan de lado la determinación y dan paso a un estado de "falta de certeza», que deconstruye la narrativa de una relación semántica equivalente y estable entre textos, como plantea Pym (2010, p. 120). En el caso de Jiang (2020), su investigación se enmarca mediante conceptos como «reescritura», «continuum of retranslations», «transmedial translation series» y también el concepto bajtiniano del «carnaval» para analizar la manera en que los fanáticos de la serie reescribieron/retradujeron distintos componentes de la serie de televisión y a partir de ello conformaron una comunidad en línea. Otro ejemplo, es el estudio de Bowman (2010), quien parte de conceptos psicoanalíticos y poscoloniales para estudiar la manera en que el doblaje y la subtitulación producen representaciones de otredad en las películas de Bruce Lee. "Certain binaries are blurred because of this fractured bilinguality. These are the very binaries that fundamentally structure and hierarchize many approaches to translation: fidelity/infidelity, primary/secondary, original/copy, authentic/construction, and so forth" (Bowman, 2010, p. 401). De esta manera, las jerarquías convencionales entre texto original y traducción, la linealidad del proceso de traducción o la función subsidiaria del traductor se deconstruyen con datos empíricos de la agencia de los traductores aficionados y de las traducciones que integran 
una narrativa queer a partir de un subtexto convencionalmente heteronormativo.

Las visiones del mundo suelen tener un correlato en el enfoque de investigación elegido (cualitativo, cuantitativo o mixto), en particular, en relación con los métodos y diseños, así como el tipo de datos que se producirán o recogerán. ${ }^{2}$ El enfoque cualitativo, denominado también «investigación naturalista» (naturalistic inquiry), se basa principalmente en el proceso para comprender las prácticas simbólicas en el marco del mundo social (Lincoln \& Guba, 2013, p. 46); por ejemplo, la exploración de productos audiovisuales para comprender los sentidos creados mediante el uso de la lengua. Los veintiocho estudios (100\%) que forman parte de esta sistematización comprenden elementos que apuntan al enfoque cualitativo, principalmente, el tipo de datos usados (material textual, textos audiovisuales, imágenes) y el método (en general, el estudio de caso). Uno de los aspectos claves de la investigación cualitativa es el carácter o dimensión textual de los datos. En el caso de los estudios sintetizados en este artículo, el trabajo con material textual es prioritario. Como se observa en la figura 8, todas las investigaciones recurren a material textual. Las investigaciones que recurren a datos cualitativos varios, como imágenes estáticas o en movimiento (Bernabo, 2017; Boellstorff, 2003; Bowman, 2010; Bradbury-Rance, 2017; Jiang, 2020; Kinloch, 2011; Mazdon, 2000), se enfocan en la dimensión textual de dichos datos con fines hermenéuticos de reconocer su función en la creación de significados y de acceder, mediante estos, a la diferencia y al reconocimiento de representaciones hegemónicas. En el caso de las investigaciones que usan datos cuantitativos (Díaz Pérez, 2018; Ranzato, 2015; Sandrelli, 2016; Valdeón, 2010; Villanueva Jordán, 2019), dichos datos no son parte de diseños mixtos, sino que sirven para describir los resultados del procesamiento de los datos ${ }^{3}$. Es decir, los resultados que tienen forma numérica (por ejemplo,

\footnotetext{
${ }^{2}$ En su libro sobre metodología de la investigación en traducción e interpreración, Saldanha y O'Brien (2103) platean que: «The approach to take to one's research should be determined by the research question(s) and how best it/they might be addressed. The quantitative approach is associated with the positivist epistemological position we mentioned earlier while a qualitative approach is generally associated with the interpretivist position» (p. 22). No obstante, antes de la problematización y la elaboración de las preguntas y objetivos de investigación (que podrían tener fraseos específicos de acuerdo con el enfoque), se encuentran los supuestos epistemológicos mediante los que el investigador o la investigadora establece su relación con el objeto o los sujetos del estudio (Creswell \& Creswell, 2018). En ese sentido, lo enunciado por Saldanha y O'Brien (2013) revela una visión del mundo pragmática —con supuestos filosóficos específicos y diferenciados del pospositivismo y el constructivismo- que permite recurrir a diseños y métodos cualitativo, cuantitativo o mixto debido a su efectividad.

${ }^{3}$ Véase el trabajo de Lewandowska-Tomaszcyk (2012) en relación con las formas de integrar el enfoque cualitativo y cuantitativo mediante distintos métodos comunes ya en la traductología.
} 
porcentajes) también se interpretan desde un posicionamiento cualitativo (Bernard \& Ryan, 2010).

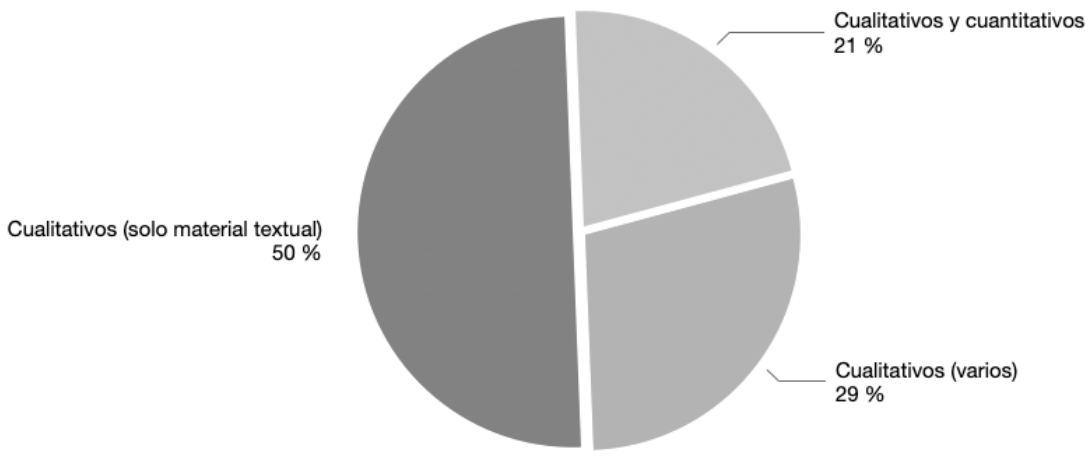

Figura 8. Tipos de datos

Fuente: elaboración propia

Si bien estos estudios tienen como base el estudio de la lengua y la traducción para comprender los procesos de representación de las identidades sexuales disidentes, resulta importante señalar que también han surgido posiciones encontradas entre las visiones del mundo constructivista y pospositivista, principalmente, en cuanto a la función de los marcos teóricos preexistentes y la teoría que emerge de los datos. Dos casos son representativos de esta disonancia entre enfoque cualitativo, la visión constructivista y el uso de la teoría. En el estudio de Turek (2012), por ejemplo, los cambios de traducción identificados por la autora en segmentos específicos, se interpretan no solo a partir de una propuesta teórica, sino que se evalúan a partir de dicha teoría.

This particular rendering may reflect an attempt to incorporate principles guiding Jean-Paul Vinay and Jean Darbelnet's theory of transposition, involving «replacing one class of words by another without changing the meaning of the message» (94). Vinay and Darbelnet assert that while French tends to be expressed in nominal expressions (as in the use of pédé), English shows a preference for verbal expressions (100). While the subtitler has tried to transpose the French and English expressions, "camp it up» in no way approximates the impact of «faggot» (Turek, 2012, p. 1024).

En la cita anterior, se usa uno de los procedimientos de Vinay y Darbelnet para evaluar la adecuación (incorporación) de una selección léxica vista en el texto meta. La noción de adecuación no tiene que ver con aspectos funcionales o comunicativos, sino con la correspondencia entre 
dato y teoría. Este posicionamiento de la teoría, como baremo de la realidad, se vincula con visiones del mundo pospositivistas y no constructivistas; asimismo, se relaciona con enfoques más cuantitativos que cualitativos. Además, resulta interesante notar que el enfoque de Vinay y Darbelnet, propuesto a finales de la década de 1950, apunta a describir «the results of what translators are presumed to do; the categories are based on no evidence of how a translator might actually get from the source to the target» (Pym, 2010, p. 13). Es decir, el propio marco traductológico usado para estudiar el producto audiovisual traducido deriva de una concepción de equivalencia principalmente prescriptiva.

Otro caso similar de función de la teoría se encuentra en la investigación de Martínez Pleguezuelos (2017). Se trata de un análisis deductivo sobre dos películas de Pedró Almodovar (Los amantes pasajeros, 2013, y La mala educación, 2004) a partir de la propuesta de Harvey (2000b, 2000a) sobre el habla camp. La finalidad de la investigación es, de acuerdo con la información del artículo, «comprobar» la propuesta teórica. Por ello, la aplicación de la teoría como punto de partida o hipótesis restringe la capacidad interpretativa del enfoque cualitativo, en la medida que los hechos semióticos o culturales se valoran a la luz de teoría preexistente que silencia o elide la comprensión del objeto de estudio en su propio contexto. Es decir, se deja de lado la noción de categorías o teorías emergentes relacionadas con la investigación naturalista. Un ejemplo alternativo, fuera del campo de la traducción, podría ser el estudio de Alejandro Yarza (1999). Se trata de una lectura inductiva del corpus de filmes de Almodóvar existentes hasta entonces. A partir de su investigación, Yarza identifica un registro camp transversal en todas las películas, no necesariamente en lo verbal, sino sobre todo en los aspectos narrativos (acciones, personajes, discursos). De esta manera, la investigación de lo camp se enfoca en el objeto de estudio, en su contexto de producción, y en las dinámicas de las que resulta una estética particular almodovariana: las fricciones entre lo contranormativo y los rezagos de una sociedad en dictadura.

Estos componentes epistemológicos de la investigación y su relación con el planteamiento metodológico no son abordados, por lo general, en la traductología (Artero \& Şerban, 2013; Lan et al., 2009). Sin embargo, es importante tener en cuenta que el análisis de los datos en un enfoque cualitativo debería considerar siempre un balance entre lo emic y lo etic. La perspectiva emic propone comprender la construcción simbólica investigada a partir de las propias experiencias de los sujetos, o del objeto y su contexto; mientras que lo etic permite evitar una sobreidentificación con la posición del informante o del objeto, así como establecer relaciones con la 
teoría y otras posiciones transculturales (Fetterman, 2008; Savin-Baden \& Major, 2013). Estas características son importantes considerando que toda traducción es un producto de una actividad interdiscursiva, en la que los significados pueden abstraerse de sus contextos, pero su ocurrencia natural no abandona dichos vínculos contextuales. Por ello, es necesario considerar que las traducciones emergen de la interacción de elementos contextuales, es decir, surgen de una cadena de operaciones de reconceptualización (Lewandowska-Tomaszczyk, 2012, p. 4).

\section{EL DISEÑO METODOLÓGICO A PARTIR DEL ESTUDIO DE CASO}

El total de estudios analizados recurren al estudio de caso como estrategia metodológica 4 . No obstante, solo un $36 \%$ (10 estudios) de los estudios analizados menciona explícitamente la estrategia metodológica (con alguna mención del "caso» o «estudio de caso»), mientras que el otro $64 \%$ (18 estudios) establecen un diseño implícito en torno a la traducción de material audiovisual (véase la figura 9). Este primer balance se relaciona con la proposición de Meyer (2016) en cuanto a la investigación en traducción e interpretación: "Studies are rarely case studies, although individual authors or specific translations, translation strategies or translation processes are studied on the basis of naturalistic data and various nonexperimental data sources» (p. 179).

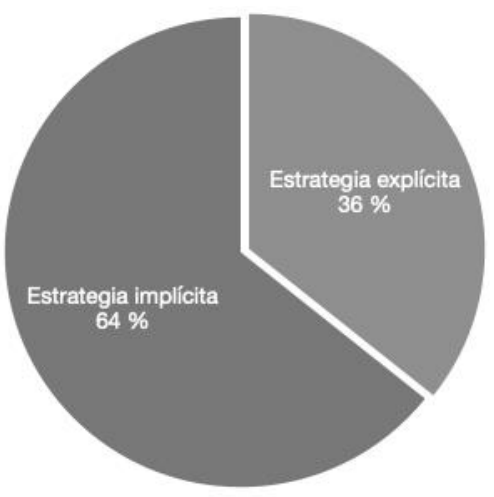

Figura 9. Estudio de caso como estrategia metodológica Fuente: elaboración propia

\footnotetext{
${ }^{4}$ Por estrategia metodológica se entiende que los estudios de caso pueden integrar distintos métodos y técnicas de producción de datos de acuerdo con las necesidades de la investigación, las características del objeto de estudio en contexto, o con fines de triangulación (Aaltio \& Heilmann, 2010; Yin, 2003).
}

Hikma 20 (2) (2021), 95 - 126 
La relación de los estudios analizados con el estudio de caso (estrategia metodológica) deriva de que estos establecen una o varias películas, series de televisión o episodios de estas como objetos de investigación suficientes o adecuados para la producción de conocimiento. Esta decisión en relación con los límites y potencialidades metodológicas de estos objetos se basa en principios del enfoque cualitativo frente a, por ejemplo, estudios cuantitativos de base estadística. Al respecto, Aalito y Heilmann (2010) elaboran lo siguiente:

The tradition of case study is different from survey study and its generalization viewpoint, since a survey study uses statistical, often multivariate, methods as a support. Case study has collapsed the generalization viewpoint of the quantitative research tradition, and has contributed to the creation of new methods in the social sciences. [...] However, cases and their analyses also form an independent methodological and methodical approach and a datagathering model for empirical social study (p. 67).

Estos aspectos «metodológicos independientes» son los que SusamSarajeva (2009) identifica como faltantes o poco claros en las investigaciones traductológicas que se llevan a cabo en programas de posgrado, pero también en la disciplina en general. «l find this vagueness surrounding the case study method in our by-now-fairly-established discipline rather problematic, especially in terms of the relationship between cases and examples, and between case studies, generalizations and theoretical frameworks» (Susam-Sarajeva, 2009, p. 37). En otras palabras, la capacidad de generalización teórica a partir de los hallazgos de los estudios de caso depende de los componentes de su diseño metodológico. Por ello, con el objetivo de comprender los diseños y análisis de los estudios incluidos en esta metasíntesis, resulta necesario identificar los criterios de selección de los casos, las unidades de análisis propuestas, así como las nociones de muestreo y generalización que constituyen los diseños metodológicos.

El primero de los componentes del diseño de investigación es la selección de los casos a partir de criterios de cantidad de casos (estudio de caso único o de casos múltiples) y de calidad del caso (típico, extremo, atípico, crítico, revelador). De los estudios analizados, un $36 \%$ (10 estudios) opta por un diseño de casos múltiples. En dichos diseños, los casos lo componen varias series de televisión, como sucede en el estudio de Sandrelli (2016) que analiza la versión norteamericana Queer as Folk, la versión de Reino Unido y la serie The $L$ Word; en otros casos, los casos corresponden a películas, como en el estudio de Bowman (2010), que aborda The Big Boss (Lo Weo, 1971) y The Way of the Dragon (Bruce Lee, 
1972). En la investigación de Ranzato (2012) se analizan tres productos audiovisuales del género dramático, aunque de distintos medios: una película para televisión (Angels in America), una serie de televisión (Six Feet Under) y una película de cine (The Boys in the Band). Otro diseño que incluye dos casos es el estudio de Villanueva Jordán (2019): la subtitulación profesional y la hecha por aficionados del programa de telerrealidad RuPaul's Drag Race.

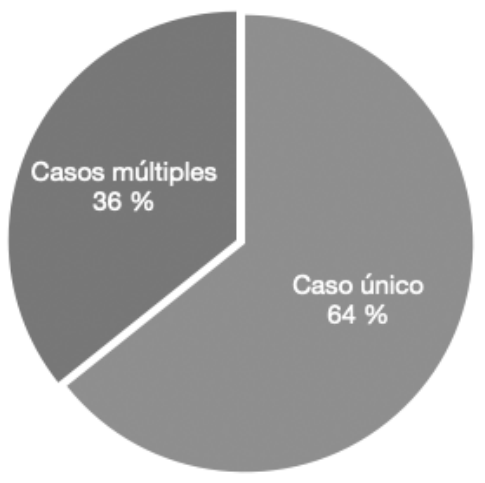

Figura 10. Diseño de estudio de caso único o casos múltiples Fuente: elaboración propia

La metodología de estudios de casos múltiples contempla un par de ventajas principales: una ventaja en cuanto al valor de verdad del que se ven favorecidos dichos estudios y la ventaja de comparación entre los hallazgos de los casos vistos de manera individual a partir de los que se pueden generar postulados teóricos inductivos (Bleijenbergh, 2010, p. 62; Yin, 2003, p. 50). Debido a que ninguno de los estudios analizados explicita el tipo de diseño metodológico usado, la primera ventaja se diluye en la exposición general del estudio. En relación con la segunda ventaja, algunos estudios sí incluyen un análisis de la transferibilidad de los resultados entre casos. Sucede así en el caso Bowman (2010) mediante el análisis individual y luego el contraste de las narrativas y de la representación de Bruce Lee en ambas películas. En el caso de Ranzato (2012), los casos se analizan de manera individual y se apunta levemente a una relación final entre los tres casos mediante la proposición de que «the translation strategies used in Italian to render the original gayspeak have suffered from the bias and preconceptions of a culture, the Italian» (Ranzato, 2012, p. 382). Por otro lado, en el caso de Sandrelli (2016), sin bien los casos se presentan de manera individual, el material textual de las series y los datos cuantitativos 
se reúnen en lo que la autora denomina «gay-themed corpus», por lo que el trabajo analítico parece apuntar, en verdad, a un estudio de caso único. Es decir, los datos de los casos iniciales se mezclan antes de analizarlos independientemente y proponer un análisis cruzado.

Los estudios que contemplan un solo caso constituyen un $64 \%$ (18 estudios) del total de las fuentes analizadas. Este dato se relaciona con el balance de Susam-Sarajeva (2001), en el que plantea que los estudios de caso múltiple eran más recurrentes en la producción académica de entonces. Por otro lado, los estudios de caso único, además de incluir una sola serie de televisión o una sola película, también pueden incluir la obra de un director siendo el criterio de autoría el que reúne el total de películas que se estudian. Esto último sucede en el caso de Díaz Pérez (2018), quien analiza la subtitulación en inglés de doce películas de Pedro Almódovar. En este estudio, el caso lo definiría Pedro Almodóvar como director, mientras que los diálogos de las películas y sus subtítulos pasan a ser parte de un corpus bilingüe que se analiza de manera integral (como sucede en el caso de Sandrelli mencionado en el párrafo anterior).

Resulta importante mencionar las investigaciones de Bernabo (2017), Bassi $(2017,2018)$, Jiang (2020), cuyos diseños se asemejan más a los propios de las ciencias sociales. Se trata de casos en los se investiga un fenómeno social contemporáneo en su contexto real, especialmente cuando los límites entre el fenómeno y su contexto no son claramente visibles (Yin, 2003). En estas investigaciones las autoras establecen un marco específico que reúne aquellos elementos del fenómeno social que serán analizados; en el caso de Bassi, los testimonios en video de sujetos que hablan de su identidad sexual o de género, mientras que, en el caso de Bernabo y Jiang, las piezas de contenido elaboradas por profesionales y aficionados en la circulación de productos audiovisuales.

Por otro lado, las investigaciones de Mazdon (2000) y BradburyRance (2017) tratan como casos independientes la versión inicial y la adaptación de las películas que son objeto de estudio. Esta mirada, más característica de los estudios de adaptación que de los estudios de TAV tradicionales, dota a ambas versiones de independencia para el análisis de sus temas, narrativas o cinematografía, sin partir de una noción de equivalencia como criterio de contraste. Como se mencionó antes, estas investigaciones se basan en una conceptualización de traducción posmoderna, relacionada con las reescrituras.

El segundo componente relacionado con el diseño metodológico son los criterios de selección de los casos - por ser casos típicos, extremos, críticos, atípicos, reveladores, longitudinales, entre otros-. El tipo de caso 
guarda relación con el tipo de pregunta de investigación y, por ende, con el alcance de los objetivos del estudio. En ese sentido, Bleijenbergh (2010) relaciona los estudios descriptivos con los casos típicos que «should give maximal information about the specific features and characteristics of a particular social phenomenon» o atípicos en los que «the social phenomenon is visible in a very pronounced way or under extreme circumstances» (p. 61). Por otro lado, en estudios exploratorios, son más pertinentes los casos críticos a partir de cuyo análisis se pueden elaborar nuevos postulados teóricos o proponer una revisión de teorías preexistentes (Bleijenbergh, 2010, p. 61). A pesar de su relevancia metodológica, esta información es poco accesible en el caso de los estudios analizados. Como se viene mencionando, los diseños metodológicos no se abordan de manera explícita, salvo alguna mención de la frase «estudio de caso» en la menor parte de las investigaciones.

La información disponible sobre los casos aparece en las secciones en las que se contextualiza la producción y recepción de los productos audiovisuales. En la mayoría de los estudios (72 \%, 20 estudios) se opta por plantear que el caso constituye un hecho atípico. Un ejemplo saliente de esta caracterización del caso es Will \& Grace, que aparece en cuatro de los estudios analizados (Martínez Pleguezuelos, 2016, 2018; Toto, 2009; Valdeón, 2010). Los autores plantean que la serie inicia en el umbral del siglo XXI, con una propuesta narrativa inclusiva a partir de la que se elaborarán nuevas representaciones sobre las identidades sexuales. No obstante, el aspecto destacado de la serie es que se la considera innovadora, en ese sentido, atípica en relación con otras series cómicas de entonces (Martínez Pleguezuelos, 2016; Valdeón, 2010).

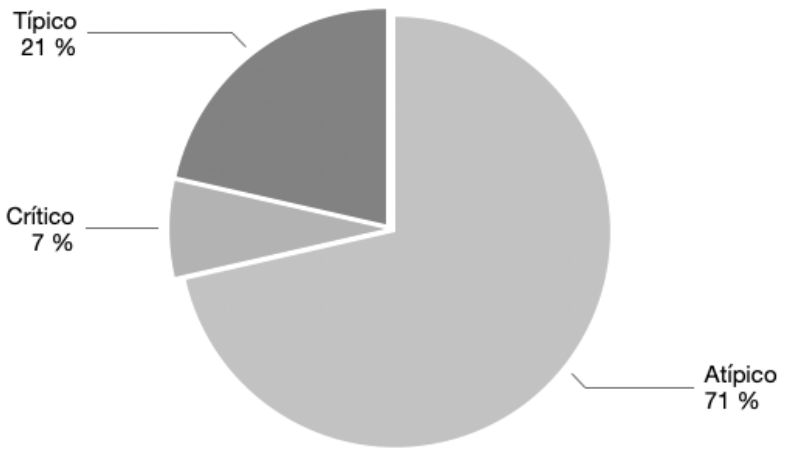

Figura 11. Tipo de caso: típico, crítico, atípico Fuente: elaboración propia

Hikma 20 (2) (2021), 95 - 126 
Con respecto a los casos típicos (21\%, 6 estudios), se puede mencionar la investigación de Ranzato (2015) en la que la serie Six Feet Under se presenta como una serie que cumple con las características narrativas y estéticas de las producciones de HBO. En el caso de la investigación de Sandrelli (2016), las series analizadas también constituyen tres casos típicos relacionados con el inicio de la representación de la diversidad sexual en los dramas de televisión. En el caso de Turek, la autora menciona que ambas películas de su estudio corresponden a un diseño muestral "representativo» (lo que puede entenderse erróneamente como un diseño estadístico); no obstante, al considerar las películas tuvieron una recepción similar al de otras películas en Estados Unidos, se entiende que se trata de casos típicos.

The two French-language films The Closet (François Veber, 2001) and My Life in Pink (Alain Berliner, 1997) ranked 37th and 70th, respectively, in gross box-office returns among all domestic and foreign gay and lesbian-themed films released in the US from 1980 to the present («Gay/Lesbian»), indicating a representative sampling of typical viewership for this genre of film (Turek, 20012, p. 1020; el subrayado es nuestro).

Bassi (2017) también recurre al concepto de representatividad cuando explica el tipo de muestreo que ha realizado en relación con el total de videos del proyecto Le cose cambiano disponibles en Youtube. No obstante, por la explicación que ofrece, se trata de un muestreo propositivo - teórico de acuerdo a las unidades de análisis que incluirá en su investigación; es decir, no se trata de un muestreo representativo con fines de generalización poblacional.

I focus on seven videos circulated on the Italian channel (created and uploaded by Alia, Filippo, Stefania, Viviana, Valentina, Anna and Davide) as representative examples of the one 123 videos presented on the localised YouTube channel. I have selected these examples because, as I explain later, their themes and specific rhetoric recur throughout the project (Bassi, 2018a, p, 237; el subrayado es nuestro).

Resulta importante mencionar que los resultados de estudios de caso típicos pueden ser transferibles a otros casos considerando las características propias de estos, así como sus nuevos contextos. Sin embargo, la generalización poblacional o estadística no es sostenible en los resultados derivados de casos. En los estudios de Ranzato (2012, 2015), por ejemplo, los casos apuntan a describir aspectos relacionados con la representación de las identidades gais mediante el doblaje en productos de ficción de cine y televisión. Por ello, los resultados podrían ser transferibles 
a nuevos casos mediante nuevas investigaciones, pero no se podrían transferir a priori a toda una población. A pesar de ello, una conclusion del estudio es que «the language of homosexuals has long remained in Italy the language of a ghetto and even today the relatively poor lexicon available is an objective obstacle even for the most unprejudiced translator » (Ranzato, 2012, p. 382). La proyección de los resultados para hablar del potencial o falta de recursos de toda una lengua en relación con la experiencia sociohistórica de un grupo humano implicaría un objeto de estudio y diseño metodológico diferente.

Los casos críticos se usan en los estudios de Jiang (2020) y Martínez Pleguezuelos (2017). En ambos estudios, las conclusiones apuntan a una revisión de categorías teóricas preexistentes a partir de los resultados de los análisis. Los casos analizados por Martínez Pleguezuelos (2017) apuntan a una actualización de las categorías descriptivas del habla camp:

[C]onsideramos necesario adoptar una perspectiva abierta hacia el uso del lenguaje y entender la clasificación que propone [Keith Harvey] como una base sobre la cual seguir analizando los rasgos del discurso camp de forma integradora, reconociendo la diferencia, y siendo capaces de representar y dar voz a las distintas identidades alojadas bajo el paraguas de la homosexualidad, sin circunscribir el discurso de las minorías sexuales a una serie limitada de rasgos específicos (p. 249).

En el caso de Jiang (2020), se apunta a una reconceptualización de la traducción y a la aplicación de enfoques hermenéuticos al análisis de las actividades de traductores aficionados en torno a productos audiovisuales y no solo a la traducción literaria:

$[T]$ his study draws attention to an expanded conceptualisation of translation which rejects the notion of translation as static, atemporal and decontextualised existence and opens up new perspectives and avenues for translation/retranslation studies. Based on hermeneutical approaches to translation, interpretative and conceptual retranslation can reach beyond the sphere of literary translation and obtain more agency through negotiation between normative and nonnormative expressions, including but not limited to sexual expressions (Jiang, 2020, p. 14).

El tercer componente del diseño metodológico de los estudios de casos es el muestral. El diseño o estrategia muestral es el componente que da al análisis del investigador una "estructura de observación» (Gillham, 2000), que contribuirá a su vez a la credibilidad y la confirmabilidad de la 
investigación ${ }^{5}$. Si bien todos los estudios analizados trabajan con categorías teóricas como sexualidad, identidad sexual o género, los criterios de muestreo solo resultan evidentes en los estudios que presentan la composición del corpus con datos numéricos (Díaz Pérez, 2018; Ranzato, 2012; Sandrelli, 2016; Valdeón, 2010; Villanueva Jordán, 2019). En las demás investigaciones ( $82 \%, 23$ estudios) se puede inferir que los autores optan por un muestreo propositivo, guiado principalmente por las categorías teóricas operativizadas mediante unidades de análisis diversas (por ejemplo, léxico, fraseología, acciones de la narrativa o temas relacionados con la sexualidad).

Sampling in case study research is largely purposeful, that is, it includes the selection of information-rich cases for in-depth study. [...] The case study approach offers flexibility in terms of the justification of sampling choice, the number of investigated cases, and sampling techniques (Fletcher \& Plakoyiannaki, 2010, p. 837).

Debido al carácter estratégico del muestreo propositivo, resulta de gran importancia que el proceso de análisis de los datos y la selección de los ejemplos sea razonado y explícito. Ello contribuye principalmente a la calidad de la investigación cualitativa, principalmente, al valor de verdad que se atribuye a las conclusiones de las investigaciones.

\section{CONCLUSIONES}

Los datos recabados en esta metasíntesis son muy esclarecedores: la gran mayoría de estudios sobre LGBTQ+ en traducción audiovisual permite inmediatamente comprender la visión epistemológica y su relación con la metodología empleada. Se trata de estudios recientes, la mayoría realizados en la última década, especialmente sobre series de televisión, centrados en pares de lenguas como el inglés y el italiano o el inglés y el español, lo que da buena cuenta de los intereses en estos temas por parte de investigadores de estas nacionalidades (un fenómeno que crece en otros pares de lenguas como se ha comprobado también), con una visión abrumadoramente constructivista del fenómeno queer, y en menor medida transformadora, lo que da cuenta claramente de los presupuestos ideológicos militantes y proactivos de los investigadores (llama la atención que no exista ni un solo estudio pospositivista para estudiar este tema), con tipos de datos eminentemente cualitativos y estudios de casos únicos, en su mayoría.

${ }^{5}$ La credibilidad implica que el análisis e interpretación de los datos corresponda a los significados y las representaciones de los casos, mientras que la confirmabilidad apunta a que la interpretación de los resultados se relacione directamente con los contenidos de los casos, sin sesgos (Guba, 1981). 
La metasíntesis ofrecida en este artículo pone al descubierto una cuestión sustancial en nuestra disciplina: el concepto mismo de «traducción». Según lo que entendamos por traducción, nuestra visión del mundo a la hora de abordar su estudio variará y, por tanto, los resultados obtenidos también variarán y serán difíciles de comparar y sobre todo de elevar a categoría más o menos universal (del universo al que representen). Es básico que se produzca un nuevo consenso sobre qué es traducción, y a su vez, que seamos capaces de definir sus límites y de repensar si este término realmente incluye todos los procesos de rescritura que se producen en el mundo audiovisual actual.

Podemos (o no) considerar las adaptaciones, narrativas transmedia, transcreaciones, remakes, etc., como traducciones y, en ese caso, las categorías de la metasíntesis, desde la visión del mundo con que se afronte el estudio, hasta los datos, las modalidades de la TAV o las estrategias metodológicas, cambiarán y se adaptarán a los objetivos de la investigación. Podemos (o no) entender la traducción desde un punto de vista pospositivo (y por tanto, más aséptico, teóricamente) o desde posiciones constructivistas o transformadoras, como las que se han revelado en este artículo, en especial, tras la digitalización y la incorporación al campo de la traducción audiovisual de los nuevos prosumidores, cuya agencia permite poner en tela de juicio la visión equivalencista y logocéntrica de la traducción que ha reinado e imperado en la práctica de la traducción y en la teoría más clásica de nuestra disciplina. Según entendamos, por tanto, el concepto de traducción, y en concreto, el de traducción audiovisual, tanto nuestra visión del mundo como nuestras estrategias metodológicas variarán en busca de unos u otros objetivos. Pero sin este consenso inicial no será posible ni entender la capacidad real de transformación de la traducción audiovisual (un fenómeno ya imparable en nuestro campo tras la digitalización) ni tampoco su esencia misma.

Las críticas de Susam-Sarajeva $(2001,2009)$ siguen siendo pertinentes una o dos décadas después. Tanto el entorno profesional de los investigadores, a los que se les exige una tesis doctoral como conditio sine qua non para su promoción o permanencia en la universidad, como la falta de consenso sobre el concepto de traducción, dan como resultado estudios adscritos parcial o totalmente a un solo paradigma, que muchas veces pretenden generalizar sus conclusiones a todo un subsistema cultural, el de los textos audiovisuales traducidos, de por sí esencialmente heterogéneo.

La utilidad de una metasíntesis como la que aquí se ofrece es, pues, poner de relieve las presuposiciones con las que se efectúan los estudios, propias de las limitaciones del sistema universitario o de la posición vital de los mismos investigadores que necesariamente deben optar por el estudio 
de un corpus manejable y abordable en un tiempo determinado, así como por la metodología que mejor se ajuste a sus objetivos. Nada de todo esto invalida -faltaría más - los resultados de las investigaciones en la TAV. Todo lo contrario. Simplemente el presente estudio nos ayuda a entenderlas en su contexto y a clasificarlas según la concepción epistemológica y metodológica del concepto de traducción que subyace a las mismas.

REFERENCIAS BIBLIOGRÁFICAS

\section{Fuentes primarias}

*Aquellos estudios incluidos en la metasíntesis y que no fueron citados en este artículo.

Asimakoulas, D. (2012). Dude (Looks like a Lady). Hijacking Transsexual Identity in the Subtitled Version of Strella by Panos Koutras. The Translator, 18(1), 47-75.

Bassi, S. (2017). Displacing LGBT. Global Englishes, Activism and Translated Sexualities. In O. Castro \& E. Ergun (Eds.), Feminist Translation Studies. Local and Transnational Perspectives (pp. 235248). Routledge Taylor \& Francis Group.

Bassi, S. (2018). The Future Is a Foreign Country. Translation and Temporal Critique in the Italian It Gets Better Project. In B. Baer \& K. Kaindl (Eds.), Queering Translation, Translating the Queer (1st ed., pp. 5871). Routledge. https://doi.org/10.4324/9781315505978-5.

Bernabo, L. E. (2017). Glee-talia: adapting Glee for an Italian audience, Critical Studies. Critical Studies in Media Communication, 1-9. https://doi.org/http://dx.doi.org/10.1080/15295036.2017.1292040.

Boellstorff, T. (2003). Dubbing culture: Indonesian gay and lesbi subjectivities and ethnography in an already globalized world. American Ethnologist, 30(2), 225-242. https://doi.org/10.1525/ae.2003.30.2.225.

*Bucaria, C. (2010). Laughing to Death: Dubbed and Subtitled Humour in Six Feet Under. In D. Chiaro (Ed.), Translation, Humour and the Media: Translation and Humour Volume 2 (pp. 222-237). Continuum.

Bowman, P. (2010). Sick man of transl-Asia: Bruce Lee and rey chow's queer cultural translation. Social Semiotics, 20(4), 393-409. https://doi.org/10.1080/10350330.2010.494393. 
Bradbury-Rance, C. (2017). The translation of desire: Queering visibility in Nathalie... and Chloe. In B. J. Epstein \& R. Gillett (Eds.), Queer in Translation. Routledge. https://doi.org/10.4324/9781315603216.

${ }^{*}$ De Marco, A. (2013). Translating Gender on Screen across Languages: The Case of Transamerica. In E. Federici \& V. Leonardi (Eds.), Bridging the Gap between Theory and Practice in Translation and Gender Studies (pp. 122-132). Cambridge Scholars Publishing.

Díaz Pérez, F. J. (2018). Language and identity representation in the English subtitles of Almodóvar's films. Cultus: The Journal of Intercultural Mediation and Communication, 11, 96-121.

*Dore, M., \& Zarrelli, I. (2018). Transfeminine Identity and HIV/AIDS in Audiovisual Translation. Dallas Buyers Club and Its Italian Subtitled Versions. In J. T. Williams Camus, C. Gómez Castro, A. Assis Rosa, \& C. Camus Camus (Eds.), Translation and Gender: Discourse Strategies to Shape Gender (pp. 59-78). Cantabria University Press.

Guo, T., \& Evans, J. (2020). Translational and transnational queer fandom in China: the fansubbing of Carol. Feminist Media Studies, 20(4), 515529. https://doi.org/10.1080/14680777.2020.1754630.

Jiang, M. (2020). Retranslation in popular culture: a multimodal extension of taboo love. The Translator, 26(1), 91-108. https://doi.org/10.1080/13556509.2019.1707385.

Leung, H. H.-S. (2016). Always in Translation. TSQ: Transgender Studies Quarterly, 3(3-4), 433-447. https://doi.org/10.1215/232892523545143.

Lewis, E. S. (2010). "This is My Girlfriend, Linda» Translating Queer Relationships in Film: A Case Study of the Subtitles for Gia and a Proposal for Developing the Field of Queer Translation Studies. In Other Words: The Journal for Literary Translators, 3, 3-22.

Martínez Pleguezuelos, A. J. (2016). Representación de la homosexualidad, identidad saliente y traducción: estudio del doblaje de Will \& Grace en español. In R. Martín Ruano \& M. C. A. Vidal Claramonte (Eds.), Traducción, medios de comunicación, opinión pública (pp. 209-226). Comares.

Martínez Pleguezuelos, A. J. (2017). Representación y traducción del camp talk en el cine de Almodóvar: los casos de «La mala educación» y «Los amantes pasajeros.» TRANS. Revista de Traductología, 21, 235-249. https://doi.org/10.24310/TRANS.2017.v0i21.3655. 
Martínez Pleguezuelos, A. J. (2018). Traducción e identidad sexual. Reescrituras audiovisuales desde la Teoría Queer (1st ed.). Editorial Comares.

*Martínez Pleguezuelos, A. J., \& González-Iglesias, J. D. (2019). Identidades presas: representación, estereotipo e interseccionalidad en la traducción de la mujer latina en Orange Is The New Black. Monografias de Traduccion e Interpretacion (MonTI), 2019(Speciallssue4), 173-198. https://doi.org/10.6035/MonTI.2019.ne4.6.

Mazdon, L. (2000). Translating Stereotypes in the Cinematic Remake. In M. Salama-Carr (Ed.), On Translating French Literature and Film II (pp. 171-182). Rodopi.

Ranzato, I. (2012). Gayspeak and Gay Subjects in Audiovisual Translation: Strategies in Italian Dubbing. Meta: Journal Des Traducteurs, 57(2), 369-384. https://doi.org/10.7202/1013951ar.

Ranzato, I. (2015). "God Forbid, a Man!»: Homosexuality in a Case of Quality TV. Between, 5(9), 1-24. https://doi.org/10.13125/2039$6597 / 1397$.

Sandrelli, A. (2016). The dubbing of gay-themed TV series in Italy: Corpusbased evidence of manipulation and censorship. Altre Modernita, 15, 124-143.

Toto, P. A. (2009). «Less about sex, more about shopping»: Will \& Grace e il linguaggio gay. In S. Petrilli \& D. Buchbinder (Eds.), Masculinities. Identità maschili e appartenenze culturali (pp. 153-158). Mimesis.

Turek, S. (2012). Gay Characters in the Margins: Gender-Based Stereotypes in Subtitled French Film. The Journal of Popular Culture, 45(5), 1020-1040. https://doi.org/10.1111/j.1540-5931.2012.00971.x.

Valdeón, R. A. (2010). Schemata, scripts and the gay issue in contemporary dubbed sitcoms. Target, 22(1), 71-93. https://doi.org/10.1075/target.22.1.05val.

*Valdeón, R. A. (2010). Dynamic versus Static Discourse: Will \& Grace and its Spanish Dubbed Version. In D. Chiaro (Ed.), Translation, Humour and the Media: Translation and Humour Volume 2 (pp. 238-249). Continuum.

Villanueva Jordán, I. (2019). «You better werk». Rasgos del camp talk en la subtitulación al español de Rupaul's Drag Race. Cadernos de 
Tradução

39(2),

$156-188$. https://doi.org/http://dx.doi.org/10.5007/2175-7968.2019v39n3p156.

Fuentes secundarias

Aaltio, I., \& Heilmann, P. (2010). Case study as a Methodological approach. In A. J. Milles, G. Durepos, \& E. Wiebe (Eds.), Encyclopedia of Case Study Research (pp. 66-76). Sage Publications.

Angelelli, C., \& Baer, B. (2016). Exploring Translation and Interpreting. In C. Angelelli \& B. Baer (Eds.), Research Translation and Interpreting (pp. 5-13). Routledge.

Artero, P., \& Şerban, A. (2013). The Status of Qualitative and Quantitative Methods of Enquiry in Translation Research: C. S. Lewis's Narnia in French - A Corpus-based Approach. Corela. Cognition, Représentation, $\quad$ Langage, 25. https://doi.org/https://doi.org/10.4000/corela.3071.

Attig, R. (2020). LGBTQ+ in translation: Emerging research from the late 2010s. Translation and Interpreting Studies. https://doi.org/https://doi.org/10.1075/tis.20104.att.

Baer, B. J., \& Kaindl, K. (2015). A Non Exhaustive Bibliography. Queering Translation, Translating the Queer.

Bernard, H. R., \& Ryan, G. W. (2010). Analyzing Qualitative Data. Systematic Approaches (1st ed.). SAGE Publications.

Bleijenbergh, I. (2010). Case Selection. In M. B. Miles, G. Durepos, \& E. Wiebe (Eds.), Encyclopedia of Case Study Research (pp. 61-63). Sage Publications.

Chaume, F. (2018). An Overview of Audiovisual Translation: Four Methodological Turns in a Mature Discipline. Journal of Audiovisual Translation, 1(1), 40-63.

Chesterman, A., \& Williams, J. (2002). The Map. A Beginner's Guide to Doing Research in Translation Studies. St Jerome Publishing.

Creswell, J. W., \& Creswell, J. D. (2018). Research Design. Qualitative, Quantitative, and Mixed Methods Approaches (5th ed.). SAGE Publications.

De Marco, M. (2012). Audiovisual Translation through a Gender Lens (1st ed.). Rodopi. 
Fetterman, D. M. (2008). Emic/Etic Distinction. In L. M. Given (Ed.), The SAGE Encyclopedia of Qualitative Research Methods (p. 249). SAGE Publications.

Fletcher, M., \& Plakoyiannaki, E. (2010). Sampling. In M. B. Miles, G. Durepos, \& E. Wiebe (Eds.), Encyclopedia of Case Study Research (pp. 837-839).

Franco Aixelá, J. (2020). BITRA (Bibliografía de Interpretación y Traducción). https://doi.org/10.14198/bitra.

Freddi, M. (2012). What AVT Can Make of Corpora: Some Findings from the Pavia Corpus of Film Dialogue. In A. Remael, P. Orero, \& M. Carroll (Eds.), Audiovisual Translation and Media Accessibility at the Crossroads. Media for All 3 (pp. 381-407). Rodopi. https://doi.org/https://doi.org/10.1163/9789401207812_021.

Gillham, B. (2000). Case Study Research Methods. Continuum.

Guba, E. G. (1981). Criteria for Assessing the Trustworthiness of Naturalistic Inquiries. ERIC/ECTJ Anual, 29(2), 75-91.

Hall, S. (1997). Representation: Cultural Representations and Signifying Practices (J. Evans, S. Hall, \& S. Nixon (eds.); 1st ed.). Sage Publications.

Harvey, K. (1998). Translating Camp Talk. The Translator, 4(2), 295-320. https://doi.org/10.1080/13556509.1998.10799024.

Harvey, K. (2000a). Gay Community, Gay Identity and the Translated Text. TTR: Traduction, Terminologie, Rédaction, 13(1), 137-165. https://doi.org/https://doi.org/10.7202/037397ar.

Harvey, K. (2000b). Translating Camp Talk. Gay Identities and Cultural Transfer. In The Translation Studies Reader (Primera, pp. 446-467). Routledge.

Karamitroglou, F. (2000). Towards a methodology for the investigation of norms in audiovisual translation: The choice between Subtitling and Revoicing in Greece. Editions Rodopi B.V.

Kinloch, D. (2011). A Queer Glaswegian Voice. In D. Asimakoulas \& M. Rogers (Eds.), Translation and Opposition. Multilingual Matters.

Lan, Y., Dong, D., \& Chiu, A. (2009). Research Trend and Methods in Translation Studies: A Comparison between Taiwanese and International Publications. Compilation and Translation Review, 2(2), 177-191. 
Lewandowska-Tomaszczyk, B. (2012). Explicit and tacit. An interplay of the quantitative and qualitative approaches to translation. In M. P. Oakes \& M. Ji (Eds.), Quantitative Methods in Corpus-Based Translation Studies. A Practical Guide to Descriptive Translation Research (pp. 334). John Benjamins Publishing Company.

Lincoln, Y. y Guba, E. (2013). The Constructivist Credo. Left Coast Press.

Martí Ferriol, J. L. (2010). Cine independiente y traducción (1st ed.). Tirant lo Blanch.

Martínez Sierra, J. J. (2008). Humor y Traducción. Los Simpson cruzan la frontera (Primera). Universitat Jaume I.

Meyer, B. (2016). Case studies. In C. Angelelli \& B. J. Baer (Eds.), Research Translation and Interpreting (pp. 177-184). Routledge.

MsCaslin, M. L. (2008). Pragmatism. In L. M. Given (Ed.), The SAGE Encyclopedia of Qualitative Research Methods (pp. 671-675). SAGE Publications.

Onwuegbuzie, A. J., \& Frels, R. (2016). 7 Steps to a Comprehensive Literature Review. A Multimodal \& Cultural Approach. SAGE Publications.

Pedersen, J. (2011). Subtitling Norms for Television (1st ed.). John Benjamins Publishing Company.

Pidduck, J. (2011). The Visible and the Sayable: the Moment and Conditions pf Hypervisibility. In F. Grandena \& C. Johnston (Eds.), Cinematic Queerness: Gayn and Lesbian Hypervisibility in Contemporary Francophone Feature Films (pp. 9-40). Peter Lang.

Pym, A. (2010). Exploring Translation Theories. Routledge.

Ranzato, I. (2016). Translating Culture Specific References On Television: The Case Of Dubbing. Routledge.

Rojo, A. (2013). Diseños y métodos de investigación en traducción. Editorial Síntesis.

Rojo, A. (2018). La investigación en traducción: un diagnóstico metodológico en diferentes ámbitos de especialidad. In A. Rojo (Ed.), La investigación en traducción. Una revisión metodológica de la disciplina (pp. 9-30). Anthropos.

Savin-Baden, M., \& Major, C. (2013). Qualitative Research. The Essential Guide to Theory and Practice (1st ed.). Routledge. 
Schensul, J. J. (2008). Methodology. In L. M. Given (Ed.), The SAGE Encyclopedia of Qualitative Research Methods (pp. 516-521). SAGE Publications.

Susam-Sarajeva, Ş. (2001). Is one case always enough? Perspectives: Studies in Translatology, 9(3), 167-176. https://doi.org/10.1080/0907676X.2001.9961415.

Susam-Sarajeva, Ş. (2009). The Case Study Research Method in Translation Studies. The Interpreter and Translator Trainer, 3(1), 3756. https://doi.org/10.1080/1750399X.2009.10798780.

Valentini, C. (2013). Phrasal verbs in Italian dubbed dialogues: A multimedia corpus-based study. Perspectives: Studies in Translatology, 21(4), 543-562. https://doi.org/10.1080/0907676X.2013.831919.

Yarza, A. (1999). Un caníbal en Madrid: la sensibilidad camp y el reciclaje de la historia en el cine de Pedro Almodóvar. Ediciones Libertarias.

Yin, R. (2003). Case Study. Design and Methods (3rd ed.). SAGE Publications. 